\title{
TITLE:
}

\section{A CHECK LIST OF THE MARINE AND FRESHWATER FISHES OF VIETNAM}

$\operatorname{AUTHOR}(\mathrm{S})$ :

Orsi, James J.

\section{CITATION:}

Orsi, James J.. A CHECK LIST OF THE MARINE AND FRESHWATER FISHES OF VIETNAM. PUBLICATIONS OF THE SETO MARINE BIOLOGICAL LABORATORY 1974, 21(3-4): 153-177

ISSUE DATE:

1974-03-30

URL:

http://hdl.handle.net/2433/175867

RIGHT: 


\title{
A GHECK LIST OF THE MARINE AND FRESHWATER FISHES OF VIETNAM
}

\author{
JAMES J. ORSI \\ Associate Marine Biologist, California Department of Fish and Game \\ Stockton, California 95205
}

I would like to express my deepest gratitude to Dr. Itiro Tomiyama for making this check list possible. Dr. Tomiyama performed most of the arduous task of checking the synonomy of the names in the list. Very few ichthyologists have the qualifications to handle such a broad range of species, including both freshwater and marine forms. I am extremely fortunate that he was kind enough to do this for me.

\section{Introduction}

The purpose of this check list is to bring together in one publication the names of all Vietnamese freshwater and marine fishes. I hope it will serve as a base for future ichthyological and general fisheries research in Vietnam. I also hope it will familiarize ichthyologists in other countries with the fauna of this area and promote exchanges of material between the Oceanographic Institute of Nhatrang and other scientific institutions.

This work was begun in 1968 when I was advisor to the Oceanographic Institute of Nhatrang for the Agency for International Development. It covers both North and South Vietnam, the Paracel Islands and the island of Poulo Condore. It is a compilation of names from many publications and records dating back to 1878 and a correction of their synonomy. Also listed are a few species I collected on the coral reefs of Nhatrang Bay and which were not previously reported from Vietnam.

The list totals 1458 species and subspecies. Those species present in the collection of the Oceanographic Institute are marked with an asterisk.

In spite of the large number of species listed the fish fauna of Vietnam is still not well known. As recently as 1963 P. Fourmanoir discovered 306 marine species new to Vietnam, and his major source of material was the commercial catch of Nhatrang. We may get an idea of the number of species still unreported by comparing the 1458 known Vietnamese species with the approximately 2200 species known from the Philippines. Vietnam should not necessarily equal the Philippines in the richness of its fauna as the island coasts of the latter country probably contain a greater variety of marine habitats than exist in Vietnam. On the other hand, Vietnam boasts a richer freshwater fauna than does the Philippines. Approximately 300 freshwater

Publ. Seto Mar. Biol. Lab., XXI (3/4), 153-177, 1974.

(Article 15) 
species have been recorded from Vietnam and the freshwater fauna can be said to be fairly well known. But since very little collecting has been done at ocean depths greater than 100 meters many new species should be present on the continental slopes.

\section{Zoogeography}

Vietnam lies on the southeast corner of Asia near the junction of the Indian and Pacific Oceans. This places it in an advantageous position to share in the faunae of several different areas. From the west, through the Straits of Malacca, trickles the fauna of the Indian Ocean. On the east, Vietnam is open to the invasion of Pacific Ocean fishes. From the north, along the continental shelf, come the coastal fishes of China and Japan. To the south, connected with Vietnam by a broad and shallow continental shelf, lies Indonesia and its rich marine fauna.

The shallow water fishes are typical of the Indo-Pacific region. Whereas, those found in depths greater than 80 meters are representative of the Japanese region. According to Chevey (1934) these northern fishes are found at the surface in Japan. Apparently, a deep southward flowing current, running parallel to the coast brings these fishes to Vietnam. During the winter monsoon, cold water from the ocean deeps, which lie close to the coast of central Vietnam, rises over the continental shelf. The summer monsoon creates currents that drive the cold waters and the fishes associated with them back off the shelf.

The freshwater fauna is heavily influenced by Vietnam's closeness to South China with its extremely rich and varied cyprinoid and catfish groups. The family Cyprinidae is Vietnam's largest, totalling 172 species. There are 7 families and 67 species of freshwater catfishes if the freshwater members of the Ariidae are counted.

The freshwater fauna is also very similar to that of Indonesia. During glacial times Vietnam and Indonesia were united by land (Krempf and Chevey, 1936).

\section{A Short History of Ichthyology in Vietnam}

The dawn of ichthyology in Vietnam came in 1876 when H.E. Sauvage published descriptions of new species of fish from the freshwaters of Vietnam. A few years later (1881) Sauvage discussed the fish fauna of Southeast Asia and again described new species from Vietnam and Cambodia.

The first major work on Vietnamese fishes was written in 1883 when Dr. Gilbert Tirant published his memoir on the fishes of the River of Hue (Tirant, 1929a, reprinted). Dr. Tirant was the French administrator for native affairs in Indochina and also mayor of Cholon, the Chinese section of Saigon. In this first list of Vietnamese fishes he described 70 species taken from the brackish waters of this river. Eight of these species were new to science. Two years later he brought out a work on the fishes of the southern part of Vietnam and Cambodia (Tirant, 1929b, reprinted). In this paper he described the biology and fishery of 221 species of sharks, rays, cyprinids, clupeids and several other groups. His list of 233 species sent to the Museum 
of Lyons, France is the first check list of Vietnamese fishes.

Another early pioneer, L. Vaillant, studied the freshwater fauna of North Vietnam during the 1890's (Vaillant, 1891).

In the early years of the twentieth century much ichthyological research was performed by the Service Océanographique des Pêches de l'Indochine (Oceanographic Service for Indochinese Fisheries). The Service was formed during the early 1920's and later became part of the Oceanographic Institute of Indochina (now that of Nhatrang) when the latter was founded in 1927.

The first check list of Vietnamese fishes intended as such was compiled in 1926 by Paul Chabanaud of the Oceanographic Service. Chabanaud's list ignored the work of earlier ichthyologists and included only species collected in the previous few years by his organization. His list contained 275 freshwater and marine species.

During the next 6 years the Oceanographic Institute continued the investigation of Vietnam's fish fauna and in 1932 the second inventory of Indochinese fishes was published (Chevey, 1932). This new list totalled 375 species, none of them included in Ghabanaud's earlier work. Thus, 650 species of fishes were known at that time.

The 1930's saw vigorous activity by the ichthyologists of the Oceanographic Institute, especially in the freshwaters of North Vietnam. "Contribution à l'etude des poissons des eaux douces Tonkinoises" (Contribution to the study of the fishes of North Vietnam) by Chevey and Lemasson (1937) is the most important work of this period. It describes 100 freshwater species, many of them new to science.

After 1939 research at the Oceanographic Institute declined drastically. The outbreak of World War II, the Japanese takeover of Indochina, and the first rumblings of the Vietnamese drive for independence hampered work in the 1940's. Between 1942 and 1947 the Institute was closed. In 1945 it was abandoned for a few months and looted with considerable loss to its collections and facilities. The French attempted to restore the Institute to its former effectiveness but the Indochinese War handicapped them greatly. Between 1952 and 1956 the French and Vietnamese jointly administered the Institute. In the latter year the Vietnamese took complete charge. But they were only partly able to replace the French scientists, all of whom left. Hence, it was not until 1961 that an ichthyological paper came out of Vietnam. This was K. Kuronuma's check list of Vietnamese fishes, a work financed by the United States Operations Mission. It contained 807 species of freshwater and marine fishes. But Kuronuma lacked many of the sources he needed to make his list complete. In 1963, ORSTOM (French Office of Scientific and Technical Overseas Research) sent P. Fourmanoir to the Oceanographic Institute. In ten months of intense activity Fourmanoir identified 306 new species of marine fishes, relying mostly on the Nhatrang market (Fourmanoir, 1965). Ten of these species were new to science.

At the present time there is little ichthyological research underway at the Institute. The war severely hindered any work, especially in the inland waters. Now that the 
war appears to have ended, the Institute can provide an ideal base for ichthyologists in Southeast Asia.

\section{List of Species}

\section{FAMILY HEXANCHIDAE-COW SHARKS}

Notorynchus cepedianus (Péron)

\section{FAMILY HETERODONTIDAE-HORN SHARKS}

Heterodontus zebra (Gray)*

3. FAMILY OREGTOLOBIDAE-GAT and CARPET SHARKS

Chiloscyllium indicum (Gmelin)*

Orectolobus japonicus Regan Ginglymostoma ferrugineum (Lesson) Stegostoma fasciatum (Hermann)*

Rhincodon typus (Smith)

4. FAMILY RHINCODONTIDAE-WHALE SHARKS

5. FAMILY LAMNIDAE-MACKEREL SHARKS

Carcharodon carcharias (Linnaeus) Isurus glaucus (Müller and Henle)

Alopias vulpinus (Bonnaterre)
6. FAMILY ALOPIIDAE-THRESHER SHARKS

\section{FAMILY CARCHARIIDAE-GRAY SHARKS}

Carcharias tricuspidatus Day

8. FAMILY SGYLIORHINIDAE-SWELL and CAT SHARKS

Atelomycterus marmoratus (Bennett)* Calliscyllium venustum Tanaka

Cephaloscyllium sufflans (Regan) Cephaloscyllium umbratile Jordan and Fowler* Halaelurus burgeri (Müller and Henle)

\section{FAMILY CARCHARINIDAE-REQUIEM SHARKS}

Aprionodon acutidens (Rüppell)

Carcharhinus dussumieri (Müller and Henle)

Carcharhinus limbatus (Müller and Henle)

Carcharhinus menissorah (Müller and Henle)*

Carcharhinus sorrah (Müller and Henle)

Hemipristis elongatus (Llunzinger)

Negogaleus microstoma (Bleeker)

Scoliodon palasorrah (Cuvier)

\section{FAMILY TRIAKIDAE--SMOOTH DOGFISHES}

Mustelus manazo Bleeker

11. FAMILY SPHYRAENIDAE-HAMMERHEAD SHARKS

Sphyrna blochi (Cuvier)*

Sphyrna mokarran (Rüppell)

12. FAMILY PRISTIDAE-SAWFISHES

Pristis cuspidatus Latham*

Pristis zijsron Bleeker

Pristiopsis microdon (Latham)

13. FAMILY RHINOBATIDAE-GUITAR FISHES

Rhina ancyclostoma Bloch and Schneider

Rhinobatos halavi (Forskål)

Rhinobatos granulatus Cuvier

Rhynchobatus djiddensis (Forskål)

14. FAMILY RAJIDAE-SKATES

Raja meerdevoorti Bleeker Carcharhinus melanopterus (Quoy and Gaimard) Carcharhinus obscurus (Lesueur) Galeocerdo cuvieri (Lesueur) Loxodon macrorhimus (Müller and Henle) Scoliodon laticaudus Müller and Henle* 


\section{FAMILY DASYATIDAE-STINGRAYS}

Dasyatis bennetti (Müller and Henle)

Dasyatis favus (Annandale)

Dasyatis imbricatus (Bloch and Schneider)*

Dasyatis kuhli (Müller and Henle)

Dasyatis zugei (Müller and Henle)*

Gymnura micrura (Bloch and Schneider)

Gymnura zonura (Bleeker)

Taeniura lymna (Forskål)*
Dasyatis bleekeri (Blyth)

Dasyatis gruveli (Ghabanaud)

Dasyatis krempfi Chabanaud

Dasyatis sephen (Forskål)

Gymnura japonica (Temminck and Schlegel)

Gymnura poecilura (Shaw)*

Himantura uarnak (Forskål)*

Urogymnus africamus (Bloch and Schneider)

16. FAMILY PLATYRHINIDAE

Zanobatus schoenleini (Müller and Henle)

17. FAMILY MYLIOBATIDAE-EAGLE RAYS

Aetobatus narinari (Euphrasen)

Aetomylaeus nichofi (Bloch and Schneider)

Aetomylaeus maculatus (Gray)

Rhinoptera javanica Müller and Henle

18. FAMILY MOBULIDAE-MANTA RAYS

Manta birostris (Walbaum) Mobula diabolus (Shaw)

19. FAMILY TORPEDINIDẢE-ELEGTRIG RAYS

Narcine lingula (Richardson)

Narcine maculata (Shaw)*

Narcine timlei (Bloch and Schneider)*

Narke dipterygia (Bloch and Schneider)*

Temera hardwicki Gray

20. FAMILY ELOPIDAE-TEN POUNDERS, GIANT HERRINGS

Elops machanata (Forskål)*

21. FAMILY MEGALOPIDAE-TARPONS

Megalops cyprinoides (Broussonet)*

22. FAMILY ALBULIDAE-LADY FISH, BONE FISH

Albula vulpes (Linnaeus)*

\section{FAMILY ANGUILLIDAE-FRESHWATER EELS}

Anguilla elphinstonei Sykes*

Anguilla marmorata Quoy and Gaimard*

Anguilla japonica Temminck and Schlegel*

Anguilla pacifica Schmitt

24. FAMILY MORINGUIDAE-WORMEELS

Moringua javanica (Kaup)*

\section{FAMILY MURAENIDAE-MORAY EELS}

Echidna delicatula (Kaup)

Echidna nebulosa $(\mathrm{Ahl})^{*}$

Echidna polyzona (Richardson)

Gymnothorax favagineus Bloch and Schneider*

Evenchelys macrurus (Bleeker)*

Gymnothorax pescadoris Jordan and Evermann

Gymnothorax meleagris (Shaw)*

Gymnothorax pikei Bliss

Gymnothorax reticularis Bloch*

Gymnothorax pictus (Ahl)*

Gymnothorax punctatofasciatus Bleeker*

Gymnothorax undulatus (Lacépède)*

Gymnothorax thrysoideus (Richardson)*

26. FAMILY MURAENESOCIDAE-FALSE CONGER EELS

Muraenesox cinereus (Forskål)*

Muraenesox talabon (Cantor)

Muraenesox talabonoides (Bleeker)

\section{FAMILY NEENCHELIDAE}

Neenchelys buitendjiki Weber and De Beaufort*

\section{FAMILY NETTASTOMIDAE_DEEP SEA EELS}

Chlopsis fierasfer Jordan and Snyder* 


\section{FAMILY GONGRIDAE-CONGER EELS}

Ariosoma anago (Temminck and Schlegel)*

Bathymyrus simus J.L.B. Smith

Conger cinereus Rüppell*

Rhynchoconger ectenurus (Jordan and Richardson)*

Uroconger lepturus (Richardson)*

\section{FAMILY OPHICHTHYIDAE-SNAKE EELS}

Brachysomophys sp.

Ophichthys apicalis (Bennett)*

Caecula sp.

Ophichthys rutiododermatoides (Bleeker)*

Pisoodonophis boro (Hamilton-Buchanan)*

Pisoodonophis cancrivorous (Richardson)

Dysomma anguillaris Barnard*

\section{FAMILY DOROSOMATIDAE-GIZZARD SHADS}

Anodontosoma chacunda (Hamilton-Buchanan)*

Clupanodon punctatus (Temminck and Schlegel)*

Anodontosoma maculatus (Richardson)*

Nematolosa nasus (Bloch)*

Clupanodon thrissa (Linnaeus)

\section{FAMILY DUSSUMIERIIDAE-ROUND HERRINGS}

Dussumeria acuta Cuvier and Valenciennes

Dussumeria hasselti Blecker*

Spratelloides delicatulus (Bennett)*

\section{FAMILY GULPEIDAE - HERRINGS, SARDINES}

Alosa platygaster (Günther)

Clupeoides lile (Cuvier and Valenciennes)*

Corica soborna Hamilton-Buchanan*

Harengula schrammi (Bleeker)*

Ilisha brachysoma (Bleeker)*

Ilisha filigera (Cuvier and Valenciennes)

Ilisha kampeni (Weber and De Beaufort)*

Ilisha melastoma (Cuvier)*

Macrura ilisha (Hamilton-Buchanan)*

Macrura macrura (Bleeker)

Macrura thibaudeani (Durand)*

Pellona ditchela Cuvier and Valenciennes*

Sardinella albella (Cuvier and Valenciennes)*

Sardinella clupeoides (Bleeker)*

Sardinella longiceps Cuvier and Valenciennes

Sardinella perforata (Cantor)*
Clupeoides borneensis Bleeker* Corica goniognathus (Bleeker)* Harengula ovalis (Bennett)* Ilisha amblyuroptera (Bleeker)* Ilisha elongata (Bennett)* Ilisha indica (Swainson)* Ilisha leschenaulti (Valenciennes)* Ilisha xanthoptera (Blceker) Macrura kelee (Cuvier)* Macrura sinensis (Linnaeus)* Opisthopterus tardoore (Guvier)* Raconda russelliana Gray*

Sardinella aurita Cuvier and Valenciennes Sardinella fimbriata (Cuvier and Valenciennes)* Sardinella melamura (Guvier)* Sardinella sirm (Walbaum)

\section{FAMILY ENGRAULIDAE-ANCHOVIES}

Coilia borneensis Bleeker* Coilia grayi Richardson* Coilia macrognathos Bleeker* Coilia rebentischi Bleeker* Setipinna breviceps (Cantor)* Setipinna phasa (Hamilton-Buchanan)

Stolephorus bataviensis Hardenberg* Stolephorus heterolobus (Rüppell)* Stolephorus tri (Bleeker) Thrissina baelama (Forskål) Thrissocles hamiltoni (Gray)* Thrissocles malabarica (Bloch)* Thrissocles setirostris (Broussonet)
Coilia dussumieri Cuvier and Valenciennes

Coilia lindmanni Bleeker*

Coilia mystus (Linnaeus)

Lycothrissa crocodilus (Blecker)* Setipinna melanochir (Bleeker)* Setipinna taty (Cuvier and Valenciennes)* Stolephorus commersoni Lacépède* Stolephorus indicus (Van Hasselt)* Stolephorus zollingeri (Bleeker)* Thrissocles dussumieri (Cuvier and Valenciennes)* Thrissocles kammalensis (Bleeker)

Thrissocles mystax (Bloch and Schneider)* 


\section{FAMILY GHIROCENTRIDAE-WOLF HERRINGS}

Chirocentrus dorab (Forskål)*

37. FAMILY OSTEOGLOSSIDAE-BONYTONGUES

Scleropages formosus (Müller and Schlegel)

38. FAMILY NOTOPTERIDAE-FEATHERBACKS

Notopterus chitala (Hamilton-Buchanan)*
39. FAMILY SALANGIDAE

Leucosoma chinensis (Osbeck)*

Notopterus notopterus (Pallas)*

Protosalanx hyalocranius (Abbott)*

Protosalanx brevirostris Pellegrin

\section{FAMILY GONOSTOMATIDAE}

Cyclothone sp.

41. FAMILY CHAULIODONTIDAE-FANG FISH

Chauliodus sloani Bloch and Schneider*.

42. FAMILY SYNODONTIDAE-LIZARD FISH

Saurida gracilis (Quoy and Gaimard)*

Synodus indicus (Day)

Saurida tumbil (Bloch)*

Synodus variegatus (Lacépède)

Synodus similis McCulloch*

Trachinocephalus myops (Bloch and Schncider)*

43. FAMILY HARPODONTIDAE

Harpodon nehereus (Hamilton-Buchanan)*

44. FAMILY MYCTOPHIDAE

Myctophum sp.

45. FAMILY ATELEOPODIDAE-DEEP SEA ATELEOPODS

Atelopus japonicus Bleeker*

46. FAMILY CHANIDAE-MILKFISH

Chanos chanos (Forskål)*

47. FAMILY CYPRINIDAE-CARP, GOLDFISH, BARBS, etc.

Acanthorhodeus taenianalis Günther*

Acheilognathus intermedius (Temminck and Schlegel)*

Albulichthys krempfi Pellegrin and Chevey*

Aristichthys nobilis (Richardson)*

Barbichthys laevis (Cuvier and Valenciennes)*

Barbus douronensis Cuvier and Valenciennes*

Carassius auratus (Linnaeus)*

Catlocarpio siamensis Boulenger*

Chela oxygaster (Cuvier and Valenciennes)*

Cirrhinus jullieni (Sauvage)*

Cirrhinus mrigala (Hamilton-Buchanan)

Crossocheilus benasi Pellegrin and Chevey*

Crossocheilus entmema (Fowler)

Crossocheilus oblongus (Cuvier and Valenciennes)

Ctenopharyngodon idellus (Cuvier and Valenciennes)*

Culter flavipinnis Tirant

Cyclocheilichthys armatus (Cuvier and Valenciennes)*

Cyclocheilichthys enoplos (Blecker)*

Cyclocheilichthys kontumensis Chevey*

Cyclocheilichthys mekongensis Fowler*

Cyclocheilichthys repasson (Bleeker)*
Acanthorhodeus tonkinensis Vaillant*

Albulichthys albuloides (Bleeker)*

Amblyrhynchichthys truncatus (Bleeker)*

Balatiocheilus melanopterus (Bleeker)*

Barbus deauratus Guvier and Valenciennes

Barbus krempfi Pellegrin and Chevey*

Catla catla (Hamilton-Buchanan)

Chela hypophthalmus Bleeker

Cirrhinus auralus (Sauvage)*

Cirrhinus microlepis (Sauvage)

Cosmocheilus pellegrini Durand*

Crossocheilus elongatus Pellegrin and Chevey*

Crossocheilus latius (Hamilton-Buchanan)

Crossocheilus reba (Hamilton-Buchanan)

Culter brevicauda Günther*

Cyclocheilichthys apogon (Cuvier and Valenciennes)*

Cyclocheilichthys enoploides (Tirant)*

Cyclocheilichthys iridescens Nichols and Pope*

Cyclocheilichthys lineatus (Popta)*

Cyclocheilichthys microstoma Pellegrin and Chevey

Cyclocheilichthys rubrepinnis microcephala

Pellegrin and Chevey 
Cyclocheilichthys siaja (Bleeker)

Cyprinus carpio multitaeniata Pellegrin and Chevey*

Dangila cuvieri Cuvier and Valenciennes*

Danio albolineatus (Blyth)

Elopichthys bambusa (Richardson)*

Erythroculter pseudobrevicaudata Nichols and Pope*

Esomus danrica (Hamilton-Buchanan)*

Garra caudofasciata (Pellegrin and Chevey)*

Garra orientalis Nichols*

Gnathopogon atromaculatus Nichols and Pope

Hemibarbus labeo (Pallas)*

Hemiculter clupeoides Nichols*

Hemiculter krempfi Pellegrin and Chevey*

Hemiculter serracanthus Nichols and Pope*

Hypophthalmichthys molitrix (Cuvier and Valenciennes)*

Labeo collaris Nichols and Pope*

Labeo erythrura Fowler

Labeo pleurotaenia (Bleeker)

Labeo xanthogenys Pellegrin and Chevey*

Labiobarbus leptocheilus (Guvier and Valenciennes)*

Labiobarbus siamensis (Sauvage)*

Leptobarbus melanotaenia Boulenger*

Leptobarbus tchangi Pellegrin and Chevey

Luciobrama longiceps Pellegrin

Luciosoma setigerum (Cuvier and Valenciennes)*

Megalobrama affinis (Vaillant)*

Megalobrama melrosei Nichols and Pope*

Mylopharyngodon aethiops (Basilewsky)*

Onychostoma laticeps Günther*

Onychostoma macracanthus Pellegrin and Chevey*

Opsarichthys elegans Pellegrin and Chevey*

Opsarichthys uncirostris (Temminck and Schlegel)

Osteochilus borneensis (Bleeker)

Osteochilus melanopleura (Bleeker)*

Osteochilus salsburyi Nichols and Pope*

Osteochilus simus (Sauvage)

Osteochilus spilura (Bleeker)

Osteochilus vittatus (Cuvier and Valenciennes)*

Oxygaster siamensis (Günther)*

Paralaubuca stigmabrachium (Fowler)*

Phoxinus lagowski Dybowski*

Pseudogyrinocheilus prochilus (Sauvage and de

Thiersant)*

Puntioplites proctozysron (Bleeker)*

Puntius annamensis Pellegrin and Chevey

Puntius aurotaeniatus (Tirant)

Puntius bramoides (Cuvier and Valenciennes)*

Puntius bulu (Bleeker)*

Puntius elongatus (Scale)
Cyprinus carpio Linnaeus*

(Hybrid) Cuprinus carpio $\times$ Carassius auratus*

Dangila fasciata Bleeker

Danio rheinarti Tirant

Erythroculter erythropterus (Basilewsky)

Erythroculter recurviceps (Richardson)

Garra bourreti (Pellegrin)*

Garra gracilis (Pellegrin and Chevey)*

Garra poilanei Petit and Tchang

Hampala macrolepidota (Cuvier and Valenciennes)*

Hemiculter balnei (Sauvage)*

Hemiculter jabouillei Chevey*

Hemiculter leucisculus (Basilewsky)*

Hypophthalmichthys harmandi Sauvage*

Labeo chrysophekadion (Bleeker)*

Labeo erythropterus Cuvier and Valenciennes*

Labeo molitorella (Cuvier and Valenciennes)

Labeo pruol Tirant

Labeobarbus brevifilis Peters

Labiobarbus lineatus (Sauvage)

Labiobarbus spilopleura (H.M. Smith)*

Leptobarbux rubripinnis (Fowler)

Lobocheilus pierrei Sauvage

Luciobrama macrocephalus (Lacépède)*

Macrochirichthys macrochirus (Cuvier and Valenciennes)*

Megalobrama bramula (Cuvier and Valenciennes)

Megalobrama terminalis (Richardson)*

Ochetobius elongatus (Kner)*

Onychostoma leptura (Boulenger)*

Onychostoma ovalis Pellegrin and Chevey

Opsarichthys hainanensis Nichols and Pope

Osteochilus barbatula (Sauvage)

Osteochilus hasselti (Cuvier and Valenciennes)*

Osteochilus melanopterus Tirant

Osteochilus schlegeli (Bleeker)

Osteochilus spilopleura Fowler

Osteochilus triporus (Bleeker)

Oxygaster anomalurus Van Hasselt*

Parabramis bramula (Cuvier and Valenciennes)*

Paralaubuca typus Bleeker*

Probarbus jullieni Sauvage*

Pseudorasbora parva (Temminck and Schlegel)*

Puntius altus (Günther)*

Puntius aureus (Tirant)*

Puntius binotatus (Cuvier and Valenciennes)*

Puntius brevis (Bleeker)*

Puntius chola (Hamilton-Buchanan)*

Puntius hainanensis (Lohberger)* 
Puntius javanicus (Bleeker)*

Puntius orphoides (Cuvier and Valenciennes)*

Puntius platysoma (Bleeker)

Rasbora argyrotaenia (Bleeker)

Rasbora daniconius (Hamilton-Buchanan)

Rasbora paviei Tirant

Rasborichthys helfrichi (Bleeker)

Sarcocheilichthys hainanensis Nichols and Pope*

Spinibarbus macracanthus Pellegrin and Chevey

Squaliobarbus annamiticus Tirant

Squaliobarbus curriculus (Richardson)*

Toxabramis houdemeri abbreviata Pellegrin

Tylognathus fowleri Pellegrin and Chevey

Varicorhinus dyocheilus (McClelland)*

Varicorhinus lemassoni Pellegrin and Chevey

Xenocheilichthys loppei Durand*

Xenocypris nitidus Garman*
Puntius laoensis (Günther)

Puntius pierrei Sauvage

Puntius strigatus (Boulenger)

Rasbora aurotaenia Tirant

Rasbora lateristriata (Bleeker)

Rasbora reticulata Weber and De Beaufort

Rasborinus lineatus (Pellegrin)*

Spinibarbus denticulatus (Oshima)*

Spinibarbus nigrodorsalis Oshima*

Squaliobarbus caudalis Sauvage

Toxabramis houdemeri Pellegrin

Tylognathus falcifer (Cuvier and Valenciennes)*

Tylognathus hispidus (Cuvier and

Valenciennes)

Varicorhinus graffeuilli Pellegrin and Chevey*

Varicorhinus tonkinensis Pellegrin and Chevey*

Xenocypris macrolepis Bleeker

Zacco temmincki (Temminck and Schlegel)*

48. FAMILY GYRINOCHEILIDAE

Gyrinocheilus aymonieri (Tirant)

Gyrinocheilus pennocki (Fowler)*

49. FAMILY HOMALOPTERIDAE

Sinogastromyzon tonkinensis Pellegrin and Chevey Swellia lineolata (Cuvier and Valenciennes)*

50. FAMILY COBITIDAE-LOACHES

Acanthopsis choirorhynchus (Bleeker)*

Barbatula toni fowleri Nichols*

Botia hymenophysa (Blecker)*

Botia modesta Bleeker*

Botia morleti Tirant

Misgurnus fossilis anguillicaudatus (Cantor)

Cobitis taenia dolichorhynchus Nichols*

Nemacheilus pulcher taeniata Pellegrin and Chevey

Misgurnus mizolepis Günther

Nemacheilus spiloptera (Cuvier and Valenciennes)

Nemacheilus spiloptera nasifilis Pellegrin

\section{FAMILY BAGRIDAE-FRESHWATER CATFISH}

Bagroides macracanthus Bleeker*

Heterobagrus bocourti Bleeker*

Leiocassis poecilopterus (Cuvier and Valenciennes)

Mystus chinensis (Steindachner)*

Mystus micracanthus (Bleeker)*

Mystus vittatus (Bloch)*

Mystus wycki (Bleeker)*

Pseudobagrus fulvidraco (Richardson)*

Pseudobagrus virgatus (Oshima)*

\section{FAMILY SILURIDAE-FRESHWATER CATFISH}

Callichrous bimaculatus (Bleeker)*

Callichrous pabo (Hamilton-Buchanan)

Cryptopterus apogon (Bleeker)*

Cryptopterus cheveyi Durand*

Cryptopterus micronema (Bleeker)

Parasilurus asotus (Linnaeus)*
Bagroides macropterus Bleeker*

Leiocassis albicollaris Fowler*

Leiocassis siamensis Regan

Mystus gulio (Hamilton-Buchanan)*

Mystus nemurus (Cuvier and Valenciennes)*

Mystus wolffi (Bleeker)*

Pseudecheneis paviei Vaillant

Pseudobagrus intermedius Nichols and Pope*
Callichrous macrophthalmus Blyth*

Cranoglanis sinensis Peters*

Cryptopterus bicirrhis (Cuvier and

Valenciennes)*

Cryptopterus cryptopterus (Bleeker)*

Cryptopterus schilbeides (Bleeker)*

Parasilurus cochinchinensis (Cuvier and Valenciennes) 
Penesilurus.bokorensis Pellegrin and Chevey Wallago attu (Bloch and Schneider)*
Silurodes hypophthalmus (Bleeker)*

Wallago leeri Bleeker

\section{FAMILY SCHILBEIDAE-SCHILBEID CATFISHES}

Helicophagus waandersi Bleeker*

Pangasius beani H.M. Smith

Pangasius larnaudi Bocourt*

Pangasius micronema Bleeker

Pangasius pangasius (Hamilton-Buchanan)*

Pangasius sutchi Fowler*

Pteropangasius cultratus (H.M. Smith)*

\section{FAMILY SISORIDAE}

Amblyceps mangois (Hamilton-Buchanan)

Glyptosternon hainanensis Nichols and Pope
Pangasius altifrons Durand*

Pangasius dezwaani Weber and De Beaufort

Pangasius macronema Bleeker*

Pangasius nasutus (Blceker)*

Pangasius polyuranodon Bleeker*

Pangasius taeniura Fowler*

Bagarius bagarius (Hamilton-Buchanan) Pareuchiloglanis poilanei Pellegrin

55. FAMILY CLARIIDAE-FRESHWATER CATFISH

Clarias batrachus (Linnaeus)*

Clarias fuscus (Lacépède)*

Claria nsieuhofi Cuvier and Valenciennes

Clarias dussumieri Guvier and Valenciennes

Clarias melanoderma Bleeker.

\section{FAMILY ARIIDAE-SEA CATFISH}

Arius caelatus Cuvier and Valenciennes*

Arius falcarius Richardson

Arius macronotacanthus Bleeker*

Arius microcephalus Bleeker*

Arius stormi (Bleeker)*

Arius truncatus Cuvier and Valenciennes*

Arius venosus Cuvier and Valenciennes*

Hemipimelodus borneensis (Bleeker)*

Hemipimelodus daugueti Chevey*

Hemipimelodus siamensis Sauvage*

Osteogeneiosus militaris (Linnaeus)

Arius cochinchinensis Günther Arius leiotetocephalus Bleeker* Arius maculatus (Thunberg)* Arius sagor (Hamilton-Buchanan)* Arius thalassinus (Rüppell)* Arius utik Bleeker* Batracocephalus mimo (Hamilton-Buchanan)* Hemipimelodus cochlearis Fowler* Hemipimelodus macrocephalus Bleeker* Ketengus typus Blecker*

57. FAMILY PLOTOSIDAE-CATFISH EELS

Paraplotosus albilabris (Cuvier and Valenciennes)* Plotosus anguillaris (Bloch)*

Plotosus canius Hamilton-Buchanan*

58. FAMILY BATRACHOIDIDAE-FROGFISH, TOADFISH

Haplophryne gangene (Hamilton-Buchanan)*

59. FAMILY LOPHIIDAE-MONKFISH, ANGLERFISH

Lophiomus setigerus (Vahl)*

60. FAMILY ANTENNARIIDAE-FISHING-FROGS

Antennarius hispidus (Bloch and Schneider)*

Histrio histrio (Linnaeus)*

Antennarius notophthalmus Bleeker*

Phrynelox striatus (Shaw)*

61. FAMILY OGOGEPHALIDAE-HANDFISH

Halieutaea stellata (Vahl)* Malthopsis lutea Alcock*

62. FAMILY GADIDAE-CODFISH

Bregmaceros mcclellandi Thompson*

63. FAMILY BROTULIDAE

Brotula maculata Day* Brotula multibarbis Temminck and Schlegel*

Neobythites macrops Günther*

64. FAMILY CARAPIDAE-PEARL FISH

Carapus gracilis (Bleeker)

Carapus homei (Ricardson) 


\section{FAMILY EXOCOETIDAE-FLYING FISH}

Cypselurus altipennis (Cuvier and Valenciennes)*

Cypselurus atrisignis Jenkins*

Cypselurus brevis (Weber and De Beaufort)*

Cypselurus oxycephalus (Bleeker)*

Cypselurus spilopterus (Cuvier and Valenciennes)*

Exocoetus volitans Linnaeus
Cypselurus articeps (Günther)* Cypselurus bahiensis (Ranzani)* Cypselurus opisthopus (Bleeker)* Cypselurus poecilopterus (Cuvier and Valenciennes)*

Evolantia micropterus (Cuvier and Valenciennes)*

Parexocoetus brachypterus (Richardson)

66. FAMILY BELONIDAE-GARFISH, NEEDLEFISH

Ablennes hians (Cuvier and Valenciennes)

Strongylura incisa (Cuvier and Valenciennes)

Strongylura leiura (Bleeker)*

Tylosurus annamensis (Chevey and Durand)*

Strongylura strongylura (Van Hasselt)*

Tylosurus melanotus (Bleeker)*

Tylosurus crocodilus (Lesueur)*

Xenentodon canciloides (Blecker)*

\section{FAMILY HEMIRAMPHIDAE-HALF BEAKS}

Vemiramphus dussumieri Cuvier and Valenciennes*

Hemiramphus far (Forskål)*

Vemiramphus gaimardi Cuvier and Valenciennes*

Hemiramphus marginatus (Forskål)*

Hemiramphus regularis Günther

Hemiramphus georgi Cuvier and Valenciennes

Hemiramphus quoyi Cuvier and Valenciennes*

Hemiramphus sinensis Günther

Hemiramphus xanthopterus Cuvier and Valenciennes

Zenarchopterus buffoni (Cuvier and Valenciennes)

Zenarchopterus dispar (Guvier and Valenciennes)

Zenarchopterus ectuntio (Hamilton-Buchanan)*

68. FAMILY CYPRINODONTIDAE-KILLIFISHES

Aplocheilus argyrotaenia (Tirant)

Aplocheilus javanicus Bleeker

Aplocheilus celebensis Weber

Aplocheilus timorensis Weber and De Beaufort

Cyprinodon calaritanus (Bonelli)

Oryzias latipes (Temminck and Schlegel)

Panchax panchax (Hamilton-Buchanan)*

69. FAMILY ATHERINIDAE-SILVERSIDES

Allanetta bleekeri (Günther)

Allanetta valenciennesi (Bleeker)*

Pranesus pinguis (Lacépède)

70. FAMILY TRACHICHTHYIDAE

Trachichthodes sp.*

71. FAMILY HOLOCENTRIDAE-SOLDIER FISH, SQUIRREL FISH

Holocentrus caudimaculatus Rüppell*

Holocentrus diadema Lacépède*

Holocentrus sammara (Forskål)*

Myripristis kaianus Günther*

Myripristis murdjan (Forskål)

\section{FAMILY CAPROIDAE-BOAR FISH}

Antigonia macrodon (Lacépède)*

73. FAMILY VELIFERIDAE

Velifer africanus Smith*

Holocentrus cornutus Bleeker*

Holocentrus ruber (Forskål)*

Myripristis hexagonus (Lacépède)*

Myripristis melanostictus Bleeker*

Myripristis pralinius Cuvier and Valenciennes*

Antigonia rubescens (Günther)*

Velifer hypsilopterus (Bleeker)

74. FAMILY AULOSTOMIDAE-CORNET FISH

Aulostomus chinensis (Linnaeus)

75. FAMILY FISTULARIIDAE-FLUTE MOUTHS, TRUMPET FISH

Fistularia petimba Lacépède* 
76. FAMILY CENTRISGIDAE-RAZOR FISH

Aeoliscus strigatus (Günther)* Centriscus scutatus Linnaeus*

77. FAMILY SYNGNATHIDAE-PIPE FISH, SEA HORSES

Bombonia spicifer (Rüppell)

Corythoichthys fasciatus (Gray)

Hippocampus borboniensis Dumeril

Hippocampus histrix Kaup*

Hippocampus kuda Bleeker*

Hippocampus takakurai Tanaka*

Hippocampus trimaculatus Leach*

Ichthyocampus carce (Hamilton-Buchanan)

Oostethus brachyurus (Bleeker)

Syngnathoides biaculeatus (Bloch)*

Oostethus deokhatoides (Bleeker)

Syngnathus schlegeli Kaup*

Syngnathus cyanospilus Bleeker

Trachyramphus serratus (Temminck and

Schlegel)

Yozia bicoarctata (Bleeker)*

Yozia longirostris (Kaup)

78. FAMILY GHANNIDAE—SNAKE HEADS

Channa asiatica (Linnaeus)*

Channa melanoptera (Bleeker)

Channa siamensis (Günther)*

Channa maculata (Lacnpède)*

Channa micropeltes (Cuvier and Valenciennes)*

Channa gachua (Hamilton-Buchanan)

Channa striatus (Bloch)*

79. FAMILY FLUTIDAE-RICE FIELD EELS

Fluta alba (Zuiew)*

Synbranchus bengalensis (McClelland)*

80. FAMILY SCORPAENIDAE - SCORPION FISH

Amblyapistus binotatus Peters

Amblyapistus macracanthus (Bleeker)*

Amblyapistus taenianotus (Cuvier and Valenciennes)*

Apistus carinatus (Bloch and Schneider)*

Brachirus zebra (Quoy and Gaimard)*

Paracentropogon indicus (Day)*

Brachypterois serrulifer Fowler*

Paracentropogon longispinis (Cuvier and Valenciennes)*

Paracentropogon profundus J.L.B. Smith*

Parascorpaena armata (Sauvage)*

Parascorbaena bandanensis (Bleeker)*

Pterois lunulata Temminck and Schlegel*

Scorpaenodes guamensis (Quoy and Gaimard)*

Parascorpaena picta (Cuvier and Valenciennes)

Pterois volitans (Linnaeus)*

Scorpaenopsis cirrhosa (Thunberg)*

Scorpaenopsis gibbosa (Bloch and Schneider)

Scorpaenopsis leonina (Richardson)

Sebastodes fuscescens (Houttuyn)*

Vespicula trachinoides (Cuvier and

Valenciennes)

\section{FAMILY TRIGLIDAE-GURNARDS}

Lepidotrigla guentheri Hilgendorf

Lepidotrigla microptera Günther

82. FAMILY PERISTEDIIDAE

Peristedion amiscus Jordan and Starks*

Satyrichthys clavilapis Fowler

83. FAMILY SYNANGEJIDAE-STONEFISH

Choridactylus multibarbis Richardson

Erosa erosa (Langsdorf)

Inimicus cuvieri (Gray)*

Leptosynancea asteroblepa (Richardson)*

Minous oxpcephalus Bleeker

Peloropsis frondosus (Günther)*

Inimicus didactylus (Pallas)*

Minous monodactylus (Bloch and Schneider)*

Minous trachycephalus (Bleeker)*

Peloropsis uranoscopus (Bloch and Schneider)*

Synanceja horrida (Linnaeus)*

\section{FAMILY PLATYCEPHALIDAE-FLATHEADS}

Elates ransonneti (Steindachner)*

Platycephalus indicus (Linnaeus)*

Rogadius asper (Cuvier and Valenciennes)*

Thysanophrys carbunculus (Cuvier and Valenciennes)

Thysanophrys japonicus (Tilesius)

Thysanophrys pristiger (Cuvier and Valenciennes)*

Thysanophrys scaber (Linnaeus)*

Thysanophrys tuberculatus (Cuvier and Valenciennes)* 


\section{FAMILY DACTYLOPTERIDAE-FLYING GURANARDS}

Dactyloptena orientalis (Cuvier and Valenciennes)*

86. FAMILY PEGASIDAE-DRAGON FISH

Pegasus draconis Linnacus

Pegasus natans Linnacus*

Pegasus volitans Linnaeus

\section{FAMILY AMBASSIDAE-GLASSY PERGHLETS}

Ambassis commersoni Cuvier and Valenciennes Ambassis gymnocephalus (Lacépède)* Ambassis kopsi Bleeker* Ambassis thomassi Day* Ambassis ranga (Hamilton-Buchanan)* Ambassis wolff Bleeker*

\section{FAMILY SERRANIDAE-ROGK CODS, GROUPERS}

Caprodon schlegeli (Günther)*

Cephalopholis boenack (Bloch)*

Cephalopholis miniatus (Forskål)*

Cephalopholis rogaa (Forskål)*

Cephalopholis urodelus (Bloch and Schneider) Coreoperca whiteheadi Boulenger*

Diploprion bifasciatus Cuvier and Valenciennes* Epinephelus amblycephalus (Bleeker)

Epinephelus awoara (Temminck and Schlegel)* Epinephelus bontoides (Bleeker)*

Epinephelus cometae Tanaka*

Epinephelus diacanthus (Cuvier and Valenciennes)* Epinephelus epistictus (Temminck and Schlegel)

Epinephelus fasciatomaculatus (Peters)*

Epinephelus flavocaeruleus (Lacépède)

Epinephelus bata Katayama

Epinephelus kohleri Schultz

Epinephelus maculatus (Bloch)

Epinephelus merra Bloch*

Epinephelus nigropunctatus Fourmanoir*

Epinephelus rhyncolepis (Bleeker)*

Epinephelus summana (Forskål)*

Epinephelus xanthopunctatus Fourmanoir*

Lateolabrax japonicus (Cuvier and Valenciennes)*

Plectropomus leopardus (Lacépède)

Plectropomus truncatus Fowler and Bean*

Pseudanthias elongatus (Franz)*

Siniperca kwangsiensis Chong*

Trisotrops dermopterus (Temminck and Schlegel)
Cephalopholis argus Bloch and Schneider*

Cephalopholis leopardus (Lacépède)*

Cephalopholis pachycentron (Cuvier and

Valenciennes)*

Cephalopholis sonnerati (Cuvier and

Valenciennes)

Chelidoperca margaritifera Weber*

Cromileptes altivelis (Cuvier and Valenciennes)*

Epinephelus akaara (Temminck and Schlegel)

Epinephelus areolatus (Forskål)*

Epinephelus bleekeri (Vaillant and Bocourt)*

Epinephelus chlorostigma (Cuvier and

Valenciennes)*

Epinephelus corallicola (Guvier and

Valenciennes)*

Epinephelus elongatus Schultz*

Epinephelus fario (Thunberg)*

Epinephelus fasciatus (Forskål)*

Epinephelus fuscoguttatus (Forskål)

Epinephelus hoedti (Bleeker)*

Epinephelus latifasciatus (Temminck and

Schlegel)

Epinephelus megachir (Richardson)*

Epinephelus morrhua (Cuvier and Valenciennes)*

Epinephelus punctatus Fourmanoir*

Epinephelus exfasciatus (Cuvier and

Valenciennes)*

Epinephelus tauvina (Forskål)*

Grammistes sexlineatus (Thunberg)*

Lates calcarifer (Bloch)*

Plectropomus maculatus (Bloch)* ·

Psammoperca waigiensis (Cuvier and

Valenciennes)*

Siniperca chuatsi Basilewsky*

Tosana nizeai Smith and Pope

\section{FAMILY PSEUDOCHROMIDAE-DOTTYBACKS}

Dampiera melanotaenia (Bleeker)*

Pseudochromis fuscus Müller and Troschel

Pseudochromis tapeinosoma Bleeker 


\section{FAMILY PLESIOPIDAE-ROUND-HEADS}

Plesiops altivelis Steindachner

Glaucosoma burgeri Richardson*

\section{FAMILY GLAUCOSOMIDAE}

92. FAMILY THERAPONIDAE-GRUNTERS, GROAKERS

Helotes sexlineatus (Quoy and Gaimard)*

Therapon jarbua (Forskål)*

Pelates quadrilineatus (Bloch)

Therapon theraps Cuvier and Valenciennes*

Banjos banjos (Richardson)

93. FAMILY BANJOSIDAE

94. FAMILY KUHLIDAE-FLAGTAILS

Kuhlia taeniura (Guvier and Valenciennes)*

\section{FAMILY GENTRARGHIDAE-FRESHWATER BASS}

Micropterus dolomieui Lacépède (Introduced)

96. FAMILY PRIAGANTHIDAE-BULLSEYES

Priacanthus cruentatus (Lacépède)*

Priacanthus macracanthus Cuvier and Valenciennes*

Priacanthus tayenus Richardson* Pseudopriacanthus niphonitus (Cuvier and Valenciennes)*

97. FAMILY APOGONIDAE-CARDINAL FISH

Apogon aureus (Lacépède)* Apogon chrysopomus Blecker* Apogon compressus (Smith and Radcliffe)* Apogon diencaeus (Smith and Radcliffe)* Apogon enneastigma Rüppell Apogon frenatus Valenciennes* Apogon hartzfeldi Bleeker* Apogon lineatus Temminck and Schlegel* Apogon melas Bleeker* Apogon nigripinnis Cuvier and Valenciennes*

Apogon quadrifasciatus Cuvier and Valenciennes Apogon septemstriatus Günther*

Apogonichthys auritus (Guvier and Valenciennes)* Apogonichthys poecilopterus (Cuvier and Valenciennes)* Archamia zosteropora (Bleeker)*

Cheilodipterus quinquelineatus Cuvier and Valenciennes Jaydia hungi Fourmanoir*
Apogon bandanensis Bleeker* Apogon chrysotaenia Bleeker* Apogon cyanosoma Bleeker* Apogon doederleini Jordan and Snyder* Apogon fasciatus White* Apogon fuscus Quoy and Gaimard* Apogon hyalosoma Bleeker Apogon marginatus Döderlein Apogon moluccensis Valenciennes* Apogon novemfasciatus Cuvier and Valenciennes* Apogon semilineatus Temminck and Schlegel* Apogon trimaculatus Cuvier and Valenciennes Apogonichthys ellioti Day* Archamia lineolata (Cuvier and Valenciennes)* Cheilodipterus macrodon (Lacépède)* Cheilodipterus singapurensis Bleeker*

Rhabdamia gracilis (Bleeker)*

Acropoma japonicum Günther*

$$
\text { 98. FAMILY ACROPOMATIDAE }
$$

99. FAMILY SILLAGINIDAE-WHITING

Sillago boutani Pellegrin Sillago maculata Quoy and Gaimard* Sillago sihama (Forskål)*

100. FAMILY BRANCHIOSTEGIDAE-TILE FISH

Branchiostegus japonicus (Houttuyn)*

Branchiostegus sericeus Herre

Branchiostegus tollardi (Chabanaud)*

Hoplolatilus fourmanoiri J.L.B. Smith

101. FAMILY MALACANTHIDAE-FALSE WHITING

Malacanthus hoedti Bleeker* 
102. FAMILY LACTARIIDAE-WHITEFISH

Lactarius lactarius (Bloch and Schneider)*

\section{FAMILY RACHYGENTRIDAE-BLAGK KINGFISH}

Rachycentron canadus (Linnaeus)*

104. FAMILY ECHENEIDAE-SUCKERFISH, REMORAS

Echeneis naucrates Linnaeus*

Remorina albescens Temminck and Schlegel*

105. FAMILY CARANGIDAE-JAGKS, HORSE MACKERELS

Alectis ciliaris (Bloch)*

Atropus atropus (Bloch and Schneider)*

Atule kalla (Cuvier and Valenciennes)*

Atule malam (Blecker)*

Caranx armatus (Forskål)*

Caranx ferdau (Forskål)*

Caranx ignobilis (Forskål)

Caranx praeustus Bennett*

Chorinemus sanctipetri Cuvier and Valenciennes

Chorinemus tol Cuvier and Valenciennes*

Decapterus lajang Bleeker*

Elagatis bipinnulatus (Quoy and Gaimard)*

Megalaspis cordyla (Linnaeus)*

Selar crumenophthalmus (Bloch)*

Seriola dumeril (Risso)

Trachinotus bailloni (Lacépède)*

Ulula mandibularis (Macleay)*

Zonichthys nigrofasciatus (Rüppell)*
Alectis indicus (Rüppell)*

Atule djedaba (Forskål)*

Alule macrurus Bleeker

Atule mate (Cuvier and Valenciennes)*

Caranx chrysophrys Guvier and Valenciennes*

Caranx fulvoguttatus (Forskål)

Caranx malabaricus (Bloch and Schneider)*

Chorinemus lysan (Forskål)*

Chorinemus tala Cuvier and Valenciennes*

Chorinemus tolooparah (Rüppell)*

Decapterus russelli (Rüppell)*

Gnathodon speciosus (Forskål)*

Selar boops (Cuvier and Valenciennes)*

Selaroides leptolepis (Cuvier and Valenciennes)*

Seriola purpurascens Temminck and Schlegel

Trachinotus blochi (Lacépède)

Uraspis helvola (Bloch and Schneider)

\section{FAMILY FORMIONIDAE}

Formio niger (Bloch)

\section{FAMILY CORYPHAENIDAE-DOLPHINS}

Coryphaena hippurus Linnaeus*

108. FAMILY MENIDAE-MOONFISH

Mene maculata (Bloch and Schneider)*

109. FAMILY LEIOGNATHIDAE-PONY FISH

Gazza minuta (Bloch)*

Leiognathus berbis (Cuvier and Valenciennes)*

Leiognathus brevirostris (Guvier and Valenciennes)*

Leiognathus equulus (Forskål)*

Leiognathus fasciatus (Lacépède)*

Leiognathus insidiator (Bloch)*

Leiognathus ruconius (Hamilton-Buchanan)*

Leiognathus splendens (Guvier)*

110. FAMILY INERMIIDAE-RED BAIT

Dipterygonotus gruveli Chabanaud*

Dipterygonotus leucogrammicus Blleeker*

111. FAMILY CAESIONIDAE

Caesio chrysozonus Guvier and Valenciennes*

Caesio cuning (Bloch)

Caesio lunaris Cuvier and Valenciennes

Caesio xanthonotus Bleeker

Paracaesio xanthurus (Bleeker)

Pterocaesio tile (Cuvicr and Valenciennes)

Caesio coerulaureus Lacépède

Caesio diagramma Bleeker*

Caesio pulcherriumus Smith*

Mirolabrichthys tuka Herre*

Pinjalo pinjalo Bleeker*

\section{FAMILY LUTJANIDAE-SNAPPERS}

Lutjanus argentimaculatus (Forskål)*

Lutjanus bohar (Forskål)*
Lutjanus biguttatus (Cuvier and Valenciennes)*

Lutjanus chrysotaenia (Bleeker)* 
Lutjanus decussatus (Cuvier and Valenciennes)*

Lutjanus ehrenbegi (Peters)*

Lutjanus gibbus (Forskål)*

Lutjanus johni (Bloch)*

Lutjanus lemniscatus (Cuvier and Valenciennes)

Lutjanus lutjanus Bloch*

Lutjanus monostigma (Cuvier and Valenciennes)*

Lutjanus rivulatus (Guvier)*

Lutjanus russelli (Bleeker)*

Lutjanus sebae (Cuvier and Valenciennes)*

Lutjanus vaigiensis (Quoy and Gaimard)*

Macolor niger (Forskal)*

113. FAMILY ETELIDAE

Aprion virescens Cuvier and Valenciennes*

Pristipomoides filamentosus (Cuvier and Valenciennes)*

Pristipomoides typus Bleeker*

\section{FAMILY NEMIPTERIDAE-THREADFIN BREAM}

Nemipterus bleekeri (Day)*

Nemipterus japonicus (Bloch)*

Nemipterus oveni (Bleeker)*

Nemipterus upenoides (Bleeker)*

Pentapodus nemurus (Bleeker)

Scolopsis bilineatus (Bloch)*

Scolopsis ciliatus (Lacépède)*

Scolopsis inermis ('Temminck and Schlegel)*

Scolopsis monogramma (Cuvier and Valenciennes)*

Scolopsis taeniopterus (Cuvier and Valenciennes)*

Scolopsis xenochrous Günther*

\section{FAMILY LOBOTIDAE-TRIPLE TAILS}

Datnioides quadrifasciatus (Sevastianov)*

Lobotes surinamensis (Bloch)*

\section{FAMILY GERRIDAE-SILVER BIDDIES}

Gerres abbreviatus Bleeker*

Gerres fllamentosus Cuvier*

Gerres macracanthus Bleeker*

Gerres oyena (Forskål)*

Pentaprion longimanus (Cantor)*

\section{FAMILY POMADASIDAE_-GRUNTERS}

Hapalogenys kishinouyei Smith and Pope

Gaterin celebicus (Blecker)*

Gaterin chrysotaenia (Bleeker)*

Gaterin lineatus (Cuvier and Valenciennes)*

Gaterin pictus (Thunberg)

Gaterin shotaf (Forskål)
Gerres acinaces Bleeker*

Gerres lucidus Cuvier and Valenciennes

Gerres macrosoma Bleeker*

Gerres setifer Hamilton-Buchanan*

\footnotetext{
Hapalogenys mucronatus (Eydoux and Souleyet)*

Gaterin chaetodontoides (Lacépède)*

Gaterin cinctus (Temminck and Schlegel)

Gaterin niger (Guvier and Valenciennes)*

Gaterin punctatissimus (Playfair)

Pomadasys argenteus (Forskål)
} 
Pomadasys argyreus (Cuvier and Valenciennes)*

Pomadasys hasta (Bloch)*
Pomadasys auritus (Cuvier and Valenciennes)

Pomadasys maculatus (Bloch)*

\section{FAMILY LETHRINIDAE-RUDDER FISH}

Gnathodentex aurolineatus (Lacépède)

Lethrinus leutjanus (Lacépède)*

Lethrinus miniatus (Bloch and Schneider)*

Lethrinus ornatus Cuvier and Valenciennes*

Lethrinus reticulatus Guvier and Valenciennes

Monotaxis grandoculis (Forskål)*

\section{FAMILY SPARIDAE-PORGIES, SEABREAMS}

Acanthopagrus berda (Forskål)*

Acanthopagrus latus (Houttuyn)

Dentex spariformis (Ogilby)*

Pagrus major (Temminck and Schlegel)*

Sparus sarba Forskål

\section{FAMILY SGIAENIDAE-DRUMS}

Johnius belengeri (Cuvier and Valenciennes)*

Johnius cujus (Hamilton-Buchanan)

Johnius ophiceps (Alcock)*

Otolithes argenteus (Cuvier and Valenciennes)*

Otolithes ruber (Bloch and Schneider)*

Otolithoides microdon (Blecker)

Pseudosciaena amblyceps Bleeker

Pseudosciaena axillaris (Cuvier and Valenciennes)*

Pseudosciaena coibor (Hamilton-Buchanan)

Pseudosciaena goldmanni (Bleeker)

Pseudosciaena sina (Cuvier and Valenciennes)*

Sciaena russelli (Cuvier and Valenciennes)

\section{FAMILY MULLIDAE-RED MULLETS, GOATFISH}

Mulloidichthys auriflamma (Forskål)*

Mulloidichthys vanicolensis (Cuvier and Valenciennes)

Parupeneus barberinoides (Bleeker)*

Parupeneus bifasciatus (Lacépède)*

Parupeneus chrysopleuron (Temminck and Schlegel)*

Parupeneus indicus (Shaw)*

Parupeneus pleurospilus (Bleeker)*

Parupeneus porphyreus (Jenkins)

Upeneus bensasi (Temminck and Schlegel)

Upeneus moluccensis (Bleeker)*

Upeneus sundaicus (Bleeker)

Upeneus vittatus (Forskål)
Johnius carutta Bloch*

Johnius dussumieri (Cuvier and Valenciennes)*

Johnius trachycephalus (Bleeker)*

Otolithes maculatus (Cuvier and Valenciennes)*

Otolithoides biauritus (Cantor)*

Pama pama (Hamilton-Buchanan)

Pseudosciaena aneus (Bloch)*

Pseudosciaena birtwistlei (Fowler)*

Pseudosciaena diacanthus (Lacépède)*

Pseudosciaena polykladiskos (Bleeker)*

Pseudosciaena soldado (Lacépède)*

\section{FAMILY MONODACTYLIDAE-SILVER BATFISH}

Monodactylus argenteus (Linnaeus)*

Monodactylus falciformis Lacépède*

\section{FAMILY PEMPHERIDAE-SWEEPERS}

Parapriacanthus uzini (Ogilby)*

Pempheris oualensis Cuvier and Valenciennes*
Pempheris moluca Guvier and Valenciennes

Pempheris vanicolensis Cuvier and

Valenciennes* 
124. FAMILY TOXOTIDAE-ARCHER FISH

Toxotes chatareus (Hamilton-Buchanan)* Toxotes jaculator (Pallas)*

Toxotes microlepis Günther*

125. FAMILY KHYPHOSIDAE-SEA CHUBS, RUDDERFISH

Khyphosus cinerascens (Forskål)

Khyphosus lembus (Cuvier and Valenciennes)

126. FAMILY GIRELLIDAE

Protheracanthus sarissophorus (Cantor)*

127. FAMILY EPHIPPIDAE-SPADEFISH

Ephippus orbis (Bloch)*

128. FAMILY PLATACIDAE-BATFISH

Platax orbicularis (Forskål)*

129. FAMILY DREPANIDAE-SICKLE FISH

Drepane punctata (Linnaeus)*

130. FAMIIY SCATOPHAGIDAE-BUTTERFISH

Scatophagus argus (Linnaeus)*

131. FAMILY GHAETODONTIDAE-BUTTERFLY FISH

Apolemichthys trimaculatus (Cuvier and Valenciennes) Centropyge tibicen (Cuvier and Valenciennes)*

Centropyge vroliki (Bleeker)*

Chaetodon bellamaris Seale*

Chaetodon chrysurus Desjardins*

Chaetodon collare Bloch*

Chaetodon melanotus Bloch and Schneider

Chaetodon octofasciatus Bloch*

Chaetodon punctatofasciatus Guvier and Valenciennes*

Chaetodon strigangulus Gmelin

Chaetodon trifasciatus Mungo Park

Chaetodon vagabundus Linnaeus*

Chaetodontoplus mesoleucus (Bloch)*

Chelmon rostratus (Linnaeus)*

Coradion chrysozonus (Cuvier and Valenciennes)*

Forcipiger longirostris (Broussonet)

Heniochus acuminatus (Linnaeus)*

Heniochus permutatus Cuvier and Valenciennes*

Pomacanthodes annularis (Bloch)*

Pomacanthodes semicirculatus (Cuvier and

Valenciennes)*

Chaetodon auriga Forskål*

Chaetodon bennetti Cuvier and Valenciennes*

Chaetodon citrinellus Guvier and Valenciennes*

Chaetodon lineolatus Cuvier and Valenciennes*

Chaetodon mertensi Cuvier and Valenciennes*

Chaetodon ornatissiumus Cuvier and Valenciennes*

Chaetodon speculum Cuvier and Valenciennes

Chaetodon triangulum Cuvier and Valenciennes*

Chaetodon unimaculatus Bloch*

Chaetodon zanzibarensis Playfair*

Chaetodontoplus septentrionalis (Temminck and Schlegel)*

Coradion altivelis $\mathrm{McCulloch*}$

Euxiphipops sexstriatus (Cuvier and Valenciennes)

Genicanthus lamarcki (Lacépède)

Heniochus monoceros Cuvier and Valenciennes*

Parachaetodon ocellatus (Cuvier and Valenciennes)*

Pomacanthodes imperator (Bloch)*

Pygoplites diacanthus (Boddaert)

132. FAMILY NANDIDAE-LEAF FISH

Nandus nebulosus (Gray)*

Pristolepis fasciatus (Bleeker)*

133. FAMILY GICHLIDAE-CICHLIDS

Tilapia mossambica (Peters)

134. FAMILY POMACENTRIDAE CORAL REEF FISH, DEMOISELL.ES

Abudefduf aureus (Cuvier and Valenciennes)

Abudefduf bankieri (Richardson)*

Abudefduf bengalensis (Bloch)*

Abudefduf coelestinus (Cuvier and

Valenciennes)* 


\author{
Abudefduf curacao (Bloch)* \\ Abudefduf flifer (Weber)* \\ Abudefduf leucogaster (Bleeker) \\ Abudefduf vaigiensis (Quoy and Gaimard)* \\ Amphiprion ephippium (Bloch)* \\ Amphiprion polymnus (Linnaeus)* \\ Chromis bitaeniatus Fowler and Bean* \\ Chromis dimidiatus (Klunzinger)* \\ Chromis weberi Fowler and Bean* \\ Chromis xanthurus (Bleeker)* \\ Chrysiptera cyanea (Quoy and Gaimard)* \\ Chrysiptera xanthonota (Bleeker)* \\ Dascyllus marginatus Rüppell* \\ Daya jerdoni (Day)* \\ Pomacentrus coelestis Jordan and Starks \\ Pomacentrus fasciatus Cuvier and Valenciennes
}

Pomacentrus moluccensis Bleeker*

Pomacentrus nigromanus Weber*

Pomacentrus prosopotaenia Bleeker*

Pomacentrus sulphureus Klunzinger

Pomacentrus tripunctatus Cuvicr and Valenciennes

Pomacentrus violascens (Bleeker)*

\section{FAMILY CIRRHITIDAE-GURLYFINS}

Cirrhitichthys aureus (Temminck and Schlegel)*

Cirrhitus pinnulatus (Bloch and Schneider)*

Cyprinocirrhites polyactis (Bleeker)*

136. FAMILY CEPOLIDAE - SNAKEFISHES

Acanthocepola abbreviata (Cuvier and Valenciennes)* Acanthocepola cuneata Smith*

Acanthocepola krusensterni (Schlegel)

Acanthocepola mesoprion (Bleeker)

137. FAMILY MUGILIDAE-GRAY MULLETS

Crenimugil crenilabis (Forskål)*

Liza ceylonensis (Günther)*

Liza macrolepis (Smith)*

Liza oligolepis (Bleeker)*

Liza seheli (Forskål)*

Mugil affinis Günther*

Mugil cephalus Linnaeus*

Mugil dussumieri Cuvier and Valenciennes*

Mugil longimanus Günther*

Mugil speigleri Bleeker*

Mugil tade Forskå1*

\section{FAMILY SPHYRAENIDAE-BARRACUDA}

Sphyraena acutipinnis Day*

Sphyraena brachygnathus Bleeker*

Sphyraena fosteri Cuvier and Valenciennes

Sphyraena langsar Bleeker*

\section{FAMILY POLYNEMIDAE-TASSEL FISH}

Eleutheronema tetradactylum (Shaw)*

Polydactylus indicus (Shaw)*
Liza caeruleomaculatus (Lacépède)*

Liza heterochilus (Bleeker)*

Liza melinoptera (Cuvier and Valenciennes)*

Liza olivaceus (Day)

Liza vaigiensis (Quoy and Gaimard)*

Mugil borneensis Bleeker*

Mugil cunnesius Guvier and Valenciennes

Mugil kelaarti Günther

Mugil planiceps Cuvier and Valenciennes

Mugil strongylocephalus Richardson

Sphyraena barracuda (Walbaum)*

Sphyraena chrysotaenia Klunzinger*

Sphyraena jello Cuvier and Valenciennes*

Sphyraena obtusata Cuvier and Valenciennes*

Polydactplus borneensis (Bleeker)*

Polydactylus longipectoralis (Weber and De

Beaufort)* 
Polydactylus plebejus (Broussonet)*

Polydactylus sextarius Bloch and Schneider*

Polynemus paradiscus Linnaeus*

140. FAMILX LABRIDAE-WRASSES

Anampses caeruleopunctatus Rüppell*

Anampses geographicus Cuvier and Valenciennes*

Cheilinus diagramma (Lacépède)*

Cheilinus oxycephalus Bleeker*

Cheilinus trilobatus Lacépède*

Cheilo inermis (Forskål)*

Choerodon schoenleini (Cuvier and Valenciennes)*

Coris gaimardi (Quoy and Gaimard)*

Duymaeria flagellifera (Cuvier and Valenciennes)

Gomphosus tricolor Quoy and Gaimard*

Halichoeres argus (Bloch and Schneider)

Halichoeres centriquadrus (Lacépèdc)*

Halichoeres hoeveni (Bleeker)*

Halichoeres javanicus (Bleeker)*

Halichoeres leucurus (Walbaum)*

Halichoeres marginatus Rüppell*

Halichores miniatus (Cuvier and Valenciennes)

Halichoeres nigrescens (Bloch and Schneider) Halichoeres trimaculatus (Quoy and Gaimard)* Hemigymnus melapterus (Bloch)*

Hemipteronotus margaritatus Fourmanoir*

Hemipteronotus verrens Jordan and Evermann*

Iniistius dea (Temminck and Schlegel)*

Labroides dimidiatus (Cuvier and Valenciennes)*

Lepidaplois axillaris (Bennett)

Lepidaplois diana (Lacépède)*

Lepidaplois mesothorax (Bloch and Schneider)*

Novaculichthy's taeniourus (Lacépède)*

Pseudolabrus gracilis (Steindachner)

Stethojulis interrupta (Bleeker)*

Stethojulis renardi (Bleeker)*

Thalassoma hardwicki (Bennett)*

Thalassoma lunare (Linnaeus)*

Thalassoma melanochir (Bleeker)

Xiphocheilus typus Bleeker*
Anampses diadematus Rüppell

Cheilinus chlorurus (Bloch)

Cheilinus fasciatus (Bloch)*

Cheilinus rhodochrous Günther*

Cheilinus undulatus Rüpp.ll

Choerodon azurio (Jordan and Snyder)*

Cirrilabrus temmincki Bleeker*

Coris variegata (Rüppell)*

Epibulus insidiator (Pallas)*

Gomphosus varius Lacépède*

Halichoeres bimaculatus Rüppell *

Halichoeres cyanopleura (Bleeker)*

Halichoeres hyrtli (Bleeker)*

Halichoeres leparensis (Bleeker)*

Halichoeres margaritaceus (Cuvier and Valenciennes)

Halichoeres melanochir Fowler and Bean*

Halichoeres nebulosus (Cuvier and

Valenciennes)*

Halichoeres prosopeion (Bleeker)*

Hemigymnus fasciatus (Bloch)*

Hemipteronotus maculosus Fourmanoir*

Hemipteronotus pentadactylus (Linnaeus)*

Hologymnosus semidiscus (Lacépède)*

Iniistius pavo (Cuvier and Valenciennes)*

Labroides lineatus Fourmanoir*

Lepidaplois bilunulatus (Lacépède)*

Lepidaplois hirsutus (Lacépède)

Lepidaplois repditio (Quoy and Gaimard)

Pseudocheilinus hexataenia (Bleekir)*

Stethojulis axillaris (Quoy and Gaimard)*

Steihojulis kalasoma (Bleeker)

Thalassoma guentheri (Bleeker)*

Thalassoma janseni (Bleeker)*

Thalassoma lutescens (Lay and Bennett)*

Verreo oxycephalus (Bleeker)

\section{FAMILY SCARIDAE-PARROT FISH}

Calotomus spinidens (Quoy and Gaimard)

Leptoscarus vaigiensis (Quoy and Gaimard)*

Scarus blochi Cuvier and Valenciennes*

Scarus dimidiatus Bleeker*

Scarus fasciatus Cuvier and Valenciennes*

Scarus fosteri Cuvier and Valenciennes*

Scarus ghobban Forskå1*

Scarus janthochir Bleeker*

Scarus microrhinos Bleeker*

Scarus oedema (Snyder)*
Chlorurus gibbus (Rüppell)

Scarops rubroviolaceus (Bleeker)

Scarus boterersi (Snyder)*

Scarus dubius Bennett*

Scarus formosus Cuvier and Valenciennes*

Scarus frenatus Lacépède*

Scarus harid Forskål*

Scarus lepidus Jenyns*

Scarus niger Forskål*

Scarus oviceps Cuvier and Valenciennes* 
Scarus randalli Schultz*

Scarus scaber Cuvier and Valenciennes*

Scarus singaporensis Bleeker*

Scarus urbanus (J.L.B. Smith)*

Sparisoma viridis (Bonnaterre)*

142. FAMILY OPISTHOGNATHIDAE-JAW FISH

Gnathypops evermanni Jordan and Snyder

Opisthognathus castelnaui Bleeker*

\section{FAMILY MUGILOIDIDAE-GRUB FISH}

Parapercis aurantiaca Döderlein

Parapercis elongata Fourmanoir*

Parapercis hexophthalma (Cuvier and Valenciennes)*

Parapercis xanthozona (Bleeker)

\section{FAMILY URANOSCOPIDAE-STAR GAZERS}

Uranoscopus bicinctus (Temminck and Schlegel)

Uranoscopus kaianus Günther*

Uranoscopus oligolepis Bleeker

Uranoscopus guttatus Guvier and Valenciennes* Uranoscopus japonicus Houttuyn

Champsodon vorax Günther*

\section{FAMILY BLENNIDAE-BLENNIES}

Andamia heteroptera (Bleeker)*

Entomacrodus decussatus (Bleeker)

Petroscirtes annamensis Chabanaud

Petroscirtes mitratus Rüppell*

Petroscirtes variabilis Cantor

Salarias bilitonensis Bleeker

Salarias edentulus (Bloch and Schneider)

Salarias frenatus (Cuvier and Valenciennes)*
Cirripectis variolosus (Cuvier and Valenciennes)*

Entomacrodus striatus (Quoy and Gaimard)*

Petroscirtes grammistes (Cuvier and Valenciennes)

Petroscirtes rhinorhynchos Blecker*

Plagiotremus spilistius Gill

Salarias dussumieri Cuvier and Valenciennes*

Salarias fasciatus (Bloch)

Xiphasia setifer Swainson*

Congrogadus nebulatus (Bleeker)*

\section{FAMILY CALLIONYMIDAE-DRAGONETS}

Callionymus japonicus Houttuyn

Callionymus sagitta Pallas*

Dactylopus dactylopus (Guvier and Valenciennes)*

\section{FAMILY GOBIIDAE-GOBIES}

Acentrogobius balteatus (Herre)

Acentrogobius caninus (Cuvier and Valenciennes)*

Acentrogobius reichei (Bleeker)*

Amblygobius sphynx (Cuvier and Valenciennes)*

Boleophthalmus pectinirostris (Linnaeus)

Ctenogobius criniger (Cuvier and Valenciennes)* Glossogobius biocellatus (Cuvier and Valenciennes) Gobiodon erythrospilus Bleeker*

Gobius philippi Tirant

Oligolepis acutipennis (Guvier and Valenciennes)

Oplopomus oplopomus (Cuvier and Valenciennes)*
Acentrogobius bellissimus J.L.B. Smith*

Acentrogobius ornatus (Rüppell)*

Amblygobius bynoensis (Richardson)

Bathygobius fuscus (Rüppell)*

Cryptocentrus filifer (Cuvier and

Valenciennes)*

Ctenogobius zonalternans (Day)

Gnatholepis calliurus Jordan and Seale*

Gobiodon quiquestrigatus (Cuvier and Valenciennes)*

Lubricogobius ornatus Fourmanoir*

Oplopomus caninoides (Bleeker)*

Oxyurichthys microlepis (Bleeker) 
Oxyurichthys tentacularis (Cuvier and Valenciennes)

Paragobiodon echinocephalus (Rüppell)*

Periophthalmus barbarus (Linnaeus)

Pseudapocryptes lanceolatus (Bloch and Schneider)*

Waitea duque (J.L.B. Smith)*

\section{FAMILY ELEOTRIDAE-SLEEPERS}

Bostrichthys sinensis (Lacépède)*

Butis melanostigma (Bleeker)*

Eleotriodes wardi (Playfair)*

Eleotris melanosoma Bleeker*

Eviota distigma Jordan and Seale*

Oxyeleotris marmorata (Bleeker)*

Oxymetopon formosum Fourmanoir*

Philypnus chalmersi Nichols and Pope*
Parachaeturichthys polynema (Bleeker)*

Periophthalmodon schlosseri (Pallas)*

Pleurosicya sp.*

Sicyodon albus Fourmanoir

\section{FAMILY TAENIOIDIDAE-RIBBON GOBIES}

Brachyamblyopus brachysoma (Bleeker)

Taeniodes cirratus (Blyth)*

Centrotrypauchen microcephalus (Bleeker)

Trypauchen vagina Bloch and Schneider*

Butis butis (Hamilton-Buchanan)*

Eleotriodes strigatus (Broussonet)*

Eleotris fusca (Bloch and Schneider)

Eleotris oxycephala Temminck and Schlegcl*

Odontobutis obscura (Temminck and Schlegel)

Oxymetopon filamentosum Fourmanoir*

Oxymetopon typus Bleeker*

Prionobutis koilomatodon (Bleeker)*

\section{FAMILY ACANTHURIDAE-SURGEON FISH}

Acanthurus elongatus (Lacépède)*

Acanthurus matoides Cuvier and Valenciennes*

Acanthurus olivaceous Bloch and Schneider*

Acanthurus toeberi (Ahl)*

Ctenochaetus striatus (Quoy and Gaimard)*

Naso brevirostris (Cuvier and Valenciennes)*

Naso thynnoides (Cuvier and Valenciennes)

Zebrasoma flavescens (Bennett)*

Zebrasoma xanthurus (Blyth)*

\section{FAMILY ZANCLIDAE-MOORISH IDOLS}

Zanclus cornutus (Linnaeus)*

\section{FAMILY SIGANIDAE-RABBITFISH}

Siganus canaliculatus (Park)

Siganus corallinus (Cuvier and Valenciennes)*

Siganus javus (Linnaeus)*

Siganus rostratus (Cuvier and Valenciennes)

Siganus virgatus (Cuvier and Valenciennes)*

\section{FAMILY TRICHIURIDAE-CUTLASS FISH}

Trichiurus haumela (Forskål)*

Trichiurus muticus Gray

Trichiurus savala Cuvier*

\section{FAMILY SCOMBRIDAE-MACKERELS, TUNAS}

Acanthocybium solandri (Cuvier and Valenciennes)

Euthynnus affinis (Cantor)*

Gymnosarda unicolor (Rüppell)*

Pelamys sp.

Rastrelliger kanagurta (Cuvier)*

Scomber japonicus Houttuyn*
Auxis thagard (Lacépède)

Grammatorcynus bicarinatus (Quoy and

$$
\text { Gaimard)* }
$$

Katsuwonus pelamis (Linnaeus)

Rastrelliger brachysoma (Bleeker)*

Sarda orientalis (Temminck and Schlegel)

Scomberomorus cambodgiense (Durand)* 
Scomberomorus commersoni (Lacépède)*

Scomberomorus kuhli (Cuvier and Valenciennes)

Scomberomorus sinensis (Lacépède)*

Thunnus obesus (Lowe)*

Thunnus thynnus (Linnaeus)

\section{FAMILY ISTIOPHORIDAE-SAILFISH, MARLINS}

Istiophorus gladius (Broussonet)

Makaira nigricans Lacépède

\section{FAMILY NOMEIDAE}

Psenes indicus (Day)*

\section{FAMILY STROMATEIDAE_-POMFRETS}

Pampus argenteus (Euphrasen)*

Psenopsis anomala (Temminck and Schlegel)*

Pampus chinensis (Euphrasen)*

\section{FAMILY ANABANTIDAE-CLIMBING PERCH}

Anabas testudineus (Bloch)*

Macropodus cupanus (Cuvier and Valenciennes)

Osphronemus goramy Lacépède*

Trichopodus leeri (Bleeker)

Trichopsis vittatus (Cuvier and Valenciennes)

Helostoma temmincki Cuvier and Valenciennes

Macropodus opercularis (Linnaeus)*

Trichogaster pectoralis (Regan)

Trichopodus trichopterus (Pallas)

\section{FAMILY MASTACEMBELIDAE-SPINY EELS}

Macrognathus aculeatus (Bloch)*

Mastacembelus armatus favus Hora*

Mastacembelus armatus (Lacépède)*

Mastacembelus erythrotaenia Bleeker*

Mastacembelus circumcinctus Hora

\section{FAMILY PSETTODIDAE-HALIBUTS}

Psettodes erumei (Bloch and Schneider)*

\section{FAMILY BOTHIDAE-LEFT HAND FLOUNDERS}

Arnoglossus aspilus (Bleeker)

Bothus myriaster (Temminck and Schlegel)

Crossorhombus azureus (Alcock)

Grammatobothus krempfi Cihabanaud

Pseudorhombus arsius (Hamilton-Buchanan)*

Pseudorhombus elevatus Ogilby*

Pseudorhombus neglectus Bleeker

Pseudorhombus pentophthalmus Günther.
Bothus mancus (Broussonet)

Bothus ovalis Regan

Engyprosopon grandisquama (Temminck and Schlegel)*

Pseudorhombus annamensis Chabanaud

Pseudorhombus diplospilus Norman

Pseudorhombus javanicus (Bleeker)

Pseudorhombus oligodon (Bleeker)*

Tephrinectes sinensis (Lacépède)

\section{FAMILY PLEURONECTIDAE-RIGHT HAND FLOUNDERS}

Samaris cristatus Gray*

165. FAMILY SOLEIDAE-SOLES

Aesopia cornuta (Kaup)

Microbuglossus ovatus Richardson

Synaptura krempfi Durand*

Synaptura panoides Blceker*

Synaptura villosa Weber*

Zebrias zebra (Bloch)
Achiroides melanorhynchus (Bleeker)*

Pardachirus pavoninus (Lacépède)*

Synaptura orientalis (Bloch and Schneider)* Synaptura quagga (Kaup)

Typhlachirus elongatus Pellegrin and Chevey 
166. FAMILY GYNOGLOSSIDAE-TONGUE FISH

Cynoglossus arel (Bloch and Schneider)

Cynoglossus macrolepidotus (Bleeker)

Cynoglossus monopus (Bleeker)*

Cynoglossus puncticeps immaculata Pellegrin and Chevey*

Cynoglossus xyphoideus Günther*
Cynoglossus lingua Hamilton-Buchanan*

Cynoglossus microlepis (Bleeker)*

Cynoglossus pellegrini $\mathrm{Wu}$

Cynoglossus trigrammus Günther*

Paraplagusia bilineata (Bloch)

167. FAMILY TRIACANTHIDAE-SPIKE FISH

Pseudotriacanthus strigillifer (Cantor)*

Triacanthus blochi Bleeker*

Triacanthus brevirostris Temminck and Schlegel*

\section{FAMILY BALISTIDAE-TRIGGER FISH}

Abalistes stellari (Bloch and Schneider)*

Balistes vidua Solander*

Balistoides conspicillum (Bloch and Schneider)

Canthidermis maculatus (Bloch)*

Pseudobalistes flavimarginatus (Rüppell)

Rhinecanthus aculeatus (Linnaeus)*

Sufflamen bursa (Lacépède)*

Sufflamen chrysoptera (Bloch and Schneider)*
Balistes vetula Linnaeus

Balistapus undulatus (Park)*

Balistoides viridescens (Bloch and Schneider)

Odonus erythrodon (Günther)

Pseudobalistes fuscus (Bloch and Schneider)

Rhinecanthus echarpe (Lacépède)*

Sufflamen capistratus (Shaw)

\section{FAMILY MONACANTHIDAE}

Aluteres monoceros (Linnaeus)*

Chaetoderma spinosissimus (Quoy and Gaimard)

Stephanolepis setifer (Bennett)

Paramonacanthus choirocephalus (Bleeker)

Pseudaluteres nasicornis (Temminck and Schlegel)*
Aluteres scriptus (Forster)

Monacanthus chinensis (Bloch)

Paraluteres sprionurus Bleeker*

Pervagor tomentosus (Linnaeus)*

Psilocephalus barbatis (Gray)*

170. FAMILY OSTRAGIONTIDAE-TRUNK FISH, BOX FISH

Lactoria cornutus Linnaeus*

Lactoria fornasini (Bianconi)*

Ostracion tuberculatus Linnaeus*

Ostracion punctatus Bloch and Schneider

Rhynchostracion rhinorhynchus (Blceker)*

171. FAMILY TETRAODONTIDAE_PUFFER FISH

Amblyrhynchotes hypselogeneion (Bleeker)

Arothron cambodgiensis (Chabanaud)*

Arothron hispidus (Linnaeus)*

Arothron lorteli (Tirant)*

Arothron palembangensis (Bleeker)

Arothron stellatus (Bloch and Schneider)*

Canthigaster margaritatus (Rüppell)

Chonerhinus modestus (Bleeker)*

Dichotomycter fluviatilis (Hamilton-Buchanan)

Fugu niphobles (Jordan and Snyder)

Lagocephalus inermis (Temminck and Schlegel)*

Lagocephalus spadiceus (Richardson)*

Takifugu oblongus (Bloch)*
Arothron biocellatus (Tirant)

Arothron fangi (Pellegrin and Chevey)

Arothron immaculatus (Bloch and Schneider)*

Arothron nigropunctatus (Bloch and Schneider)*

Arothron reticularis (Bloch and Schneider)*

Canthigaster bennetti (Bleeker)

Canthigaster valentini (Bleeker)*

Chonerhinus naritus (Richardson)

Fugu alboplumbeus (Richardson)*

Fugu rubripes (Temminck and Schlegel)

Lagocephalus lunaris (Bloch and Schneider)*

Pleuranacanthus scleratus (Forster)*

\section{FAMILY TRIODONTIDAE}

Triodon bursarius Reinwardt

173. FAMILY DIODONTIDAE-PORCUPINE FISH

Chilomycterus orbicularis (Bloch)*

Diodon holocanthus Linnaeus*
Diodon bleekeri Günther*

Diodon hystrix Linnaeus* 


\section{REFERENGES}

Chabanaud, P. 1926. Inventaire de la faune ichthyologique de l'Indochine, premiere liste. Service Océan. des Pêches de l'Indochine, Premier Note.

1926. Aperçu sommaire sur la faune ichthyologique de la région indochinoise. Service Océan. des Pêches de l'Indochine, 4e Note.

Chevey, P. 1932. Inventaire de la faune ichthyologique de l'Indochine, deuxième liste. Inst. Océan. de l'Indochine, $19 \mathrm{e}$ Note.

1934. Révision synonymique de l'oeuvre ichthyologique de G. Tirant. Inst. Océan. de l'Indochine, 7e Note.

Chevey, 0. and M. Durand. 1945. Principaux poissons coméstibles d'Indochine. Inst. Océan. Nhatrang Service de la Marine Marchande.

Chevey, P. et J. Le Masson. 1937. Gontribution à l'étude des poissons des eaux douces tonkinoises. Inst. des Récherches Agronomiques de l'Indochine, Hanoi, Imprimerie d'Extreme Orient.

Fourmanoir, P. 1965. Liste complémentaire des poissons marins de Nhatrang. Cahiers ORSTOM, Contrib. 84.

Krempf, A. and P. Chevey. 1932. Poissons des campagnes du "de Lanessan". Travaux Inst. Océan. de l'Lndochine, 4e Memoir.

Kuronuma, K. 1961. A check list of fishes of Vietnam. Div. of Agric. and Nat. Resources, U.S. Operations Mission to Vietnam.

Loi, T.N. and N. Ghau. 1964. Les poissons d'importance commercial au Vietnam. Inst. Océan. de Nhatrang, Contrib. 79: 325-425.

Pellegrin, J. 1907. Poissons du Tonkin. Bull. Mus. Paris, 13: 499.

1934. Poissons de la région d'Hanoi (Tonkin), description d'une variété nouvelle. Bull. Soc. Zool. France, T.59 (5) : 334-337.

1936. Poissons nouveaux du Haut Laos et de l'Annam. Bull. Soc. Zool. France, T.61 (4): $243-248$.

Pellegrin, J. et P. Chevey. 1934. Poissons de Nghia Lo (Tonkin, description de quatre espèces nouvelles. Bull. Soc. Zool. France, T.59 (5): 337-343.

1936. Cyprinidés nouveaux du Tonkin. Bull. Soc. Zool. France, T.61 (1): 18-27.

1936. Poissons nouveaux ou rares du Tonkin et de l'Annam. Bull. Soc. Zool. France, T.61 (3): 219-232.

1937. Poissons d'Indochine recueillis par MM. J. DeLacours et Lowe. Description d'une espèce nouvelle. Bull. Soc. Zool. France, T.62 (5): 313-318.

- 1940. Poissons nouveaux ou rares de Cochinchine. Description de deux espèces et de deux variétés. Bull. Soc. Zool. France, T.65 (3) : 153-158.

Sauvage, H.E. 1878. Note sur quelques poissons d'espèces nouvelles provenant des eaux douces de l'Indochine. Bull. Soc. Philom. Paris, Sér. 7 (2): 233-242.

1881. Récherches sur la faune ichthyologique de l'Asie et description des espèces nouvelles de l'Indochine. Nouv. Arch. Mus. Hist. Nat. Paris, 2e Sér, Y.IV: 124-194.

- 1884. Contribution à la faune ichthyologique du Tonkin. Bull. Soc. Zool. France, Iw.

Tirant, G. 1929. Reprinted. Memoire sur les poissons de la Riviere de Hue. Service Océan. des Pêches de l'Indochine, 6e Note: 1-32.

1929. Reprinted. Note sur les poissons de la Basse-Cochinchine et du Cambodge. Service Océan. des Pêches de l'Indochine, 6e Note: 43-163.

___ 1929. Reprinted. Liste des poissons de l'Indochine envoyés par le Dr. G. Tirant, au Musée des Sciences Naturelles de Lyon. Service Océan. des Pêches de l'Indochine, 6e Note: 167-175.

Vaillant, L. 1891. Sur quelque poissons rapportés du haut Tonkin par M. Pavie. Bull. Soc. Philom., Sér. 2, T.IV: 124-194. 